\title{
INFLUENCIA DE LA PRECIPITACIÓN SOBRE LA CONCENTRACIÓN DEL RADÓN EN EL SUELO: SIMULACIÓN EN LABORATORIO Y COMPARACIÓN CON DATOS DE CAMPO
}

\author{
J. R. García-Vindas \\ Universidad de Costa Rica, Escuela de Física, Sección de Física Nuclear Aplicada. \\ Email-jrgarcia@cariari.ucr.ac.cr
}

(Recibido 21/6/1999; Aceptado 1/10/1999)

\begin{abstract}
This paper presents the most relevant results of laboratory experiences and its comparison to field data. Their objective is to better understand the precipitation effect on the radon gas emanations. The results obtained in the laboratory and the predictions of the model suggested are in completely concordance with field data. It is useful as a filter source for further data.
\end{abstract}

RESUMEN: Este trabajo presenta los resultados más relevantes de un conjunto de experimentos de laboratorio, que tienen por objetivo comprender mejor el efecto de la precipitación sobre las emanaciones gaseosas de radón. Los resultados obtenidos en el laboratorio y las predicciones del modelo propuesto, concuerdan perfectamente con los datos de campo. Los resultados son de gran utilidad a la hora de filtrar la influencia de la lluvia sobre los datos de campo.

\section{INTRODUCCIÓN}

La medida de la concentración de algunos gases en el suelo tales como el $\mathrm{Rn}, \mathrm{CO}_{2}, \mathrm{He}$, $\mathrm{H}_{2} \mathrm{~S}$, es de importancia en el monitoreo y vigilancia de los edificios volcánicos. Estos gases representan en cierta forma una señal geoquímica proveniente de las capas más profundas del suelo y evidencian los cambios que se producen en los sistemas geológicos, tales como volcanes o fallas activas. Las anomalías presentadas en las concentraciones de algunos de estos gases, como el radón pueden actuar como precursores de un evento mayor (Loría et al., 1995; King, 1992; Monnin \& Seidel, 1992). De aquí que la interpretación de la medida de la concentración de estos gases, deba estudiarse con más atención, considerando los efectos producidos por algunos parámetros exteriores sobre el transporte de los mismos en el suelo. La concentración se ve influenciada por la variabilidad de algunos factores externos tales como los parámetros atmosféricos, entre los cuales podemos citar la temperatura (Mogro-Campero \& Fleisher, 1977; Klusman \&
Jaacks, 1987; Rudakov, 1985), la presión atmosférica (Clements \& Wilkening, 1974; Rudakov, 1985b; Pinault \& Baubron, 1997), la velocidad del viento (Pearson \& Jones, 1965 y 1966; Hinkle, 1994; Cotter, 1990), la precipitación (Abbad et al., 1996; Talwani et al., 1980; Papastefanou et al., 1989; ). Sin embargo son la presión atmosférica y la precipitación, los parámetros que presentan una mayor influencia.

En zonas tropicales, como la nuestra, la variabilidad climática es bien marcada, sin embargo la presión presenta una variación casi despreciable, siendo su variación máxima del orden de 4 milibares, la cual se presenta en términos de algunas horas. Para que la variación en la presión atmosférica represente una influencia significativa en la concentración de radón o de otros gases en el suelo, es necesario que esta variación se presente en un período de tiempo largo, del orden de días. Clements \& Wilkening (1974) encuentran que, variaciones del orden del $2 \%$ en la presión atmosférica, extendidas durante varios días, producen anomalías importantes en la concentración de radón. 
Por otro, lado el efecto de la precipitación en las emanaciones gaseosas es de especial interés, ya que las variaciones de esta son significativas y bien localizadas en el tiempo. Nuestro país, por ejemplo, se caracteriza por presentar una estación húmeda o lluviosa bien marcada, la cual ocurre esencialmente en los últimos siete meses del año, por consiguiente el efecto de la precipitación no es despreciable.

El objetivo de este trabajo es mostrar la influencia de la precipitación sobre la emanación del gas radón y al mismo tiempo, proponer un modelo que explique el efecto de este parámetro sobre el transporte del gas en el suelo.

\section{METODOLOGÍA}

Para estudiar el efecto de la precipitación sobre la concentración del gas radón en el suelo, se construyó en la Universidad de Montpellier II, un dispositivo que consiste en un recipiente cilíndrico de PVC de $1 \mathrm{~m}$ de altura y $1 \mathrm{~m}$ de diámetro, abierto en los dos extremos. Este se introdujo en un agujero en el suelo al aire libre en el campus de la universidad, de manera que la parte superior sobresaliera $5 \mathrm{~cm}$ sobre el nivel del suelo. Se llenó el recipiente con una mezcla de tierra y arena para lograr un medio permeable y homogéneo. Se introdujo pechblenda, la cual es una fuente natural de radón, a tres niveles de profundidad diferentes y contenida en pequeñas bolsas plásticas permeables al radón, aproximadamente un número de cinco por nivel. Lo anterior con el fin de crear un gradiente de concentración importante en el sistema y amplificar la señal natural del suelo, tratando de conservar la uniformidad del mismo (Fig. 1).

Para realizar la medidas de la concentración de radón se instalaron dos sondas Clipperton II (Monnin \& Seidel, 1998) a profundidades diferentes. Estas sondas miden la concentración cada 20 minutos. La lluvia es simulada por medio de 4 aspersores conectados a un tubo plástico de forma circular e instalado en la parte superior del recipiente. Dos tubos de PVC de un metro de largo perforados regularmente en las paredes fueron instalados verticalmente para medir

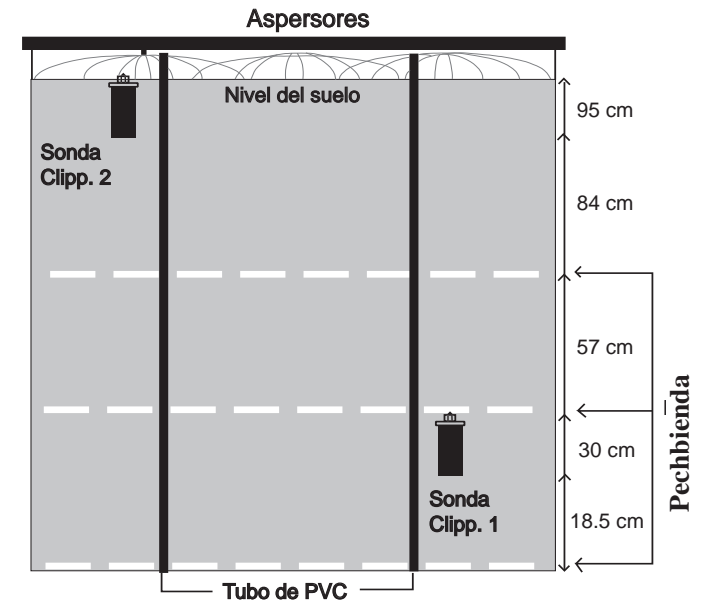

Fig.1: Dispositivo empleado para la simulación de la lluvia.

eventualmente la humedad del suelo. Estos dos tubos idénticos permanecieron cerrados durante el experimento, con el fin de no crear un camino preferencial para el gas que pudiese bajar la impedancia del sistema.

Con este dispositivo se realizaron dos experiencias consecutivas. Para la primera con el suelo inicialmente seco se dejó correr el agua durante 12 horas a un caudal de 1 litro por minuto repartido igualmente entre los cuatro aspersores. Al final de la aspersión, el suelo se encontraba completamente saturado en agua. Para iniciar la segunda experiencia (la cual es idéntica a la primera) se esperó un mes, de tal manera que el suelo retomara sus condiciones iniciales. Durante todo este tiempo de espera la concentración de radón se midió con el mismo intervalo de tiempo de 20 minutos.

\section{RESULTADOS}

Los resultados de las dos experiencias se muestran en la Figura 2. Las líneas verticales en la figura marcan el inicio y el final de la aspersión en cada experiencia. Los valores de concentración más elevados se presentan en la sonda 1 (sonda inferior del dispositivo), sin embargo las dos sondas presentan el mismo comportamiento. 
El comportamiento de ambas sondas en la segunda experiencia es igual que en la primera. La segunda experiencia comienza con un nivel de concentración un poco más alto en las dos sondas en comparación con la primera experiencia, esto se debe a que se inició con un suelo inicialmente semi-húmedo, con el fin de estudiar el efecto de la diferencia en las condiciones iniciales.

En la Figura 2 se aprecia que la concentración de radón disminuye en las dos sondas, inmediatamente después del inicio de la aspersión, para luego sufrir un aumento, el cual es más pronunciado en la sonda 1 (la inferior del dispositivo). La segunda experiencia muestra el mismo fenómeno en las dos sondas, demostrando así que el comportamiento observado en el primer caso es reproducible.

\section{DISCUSIÓN DE UN MODELO Y COMPARACIÓN DE LOS RESULTADOS CON DATOS DE CAMPO}

En los dos casos de la figura 2 la concentración de radón en las sondas disminuye al inicio de la aspersión y continua en ese estado algún tiempo después de que la aspersión finaliza. Seguidamente la concentración aumenta hasta alcanzar un máximo y luego vuelve a su estado inicial. Este comportamiento es interpretado de la manera siguiente: al principio hay dos fenómenos presentes, la disolución rápida del radón en el agua de lluvia (García-Vindas, 1999) y el transporte del radón no disuelto por el agua hacia las capas inferiores del suelo. En este ultimo caso el agua y el radón se comportan como dos fluidos

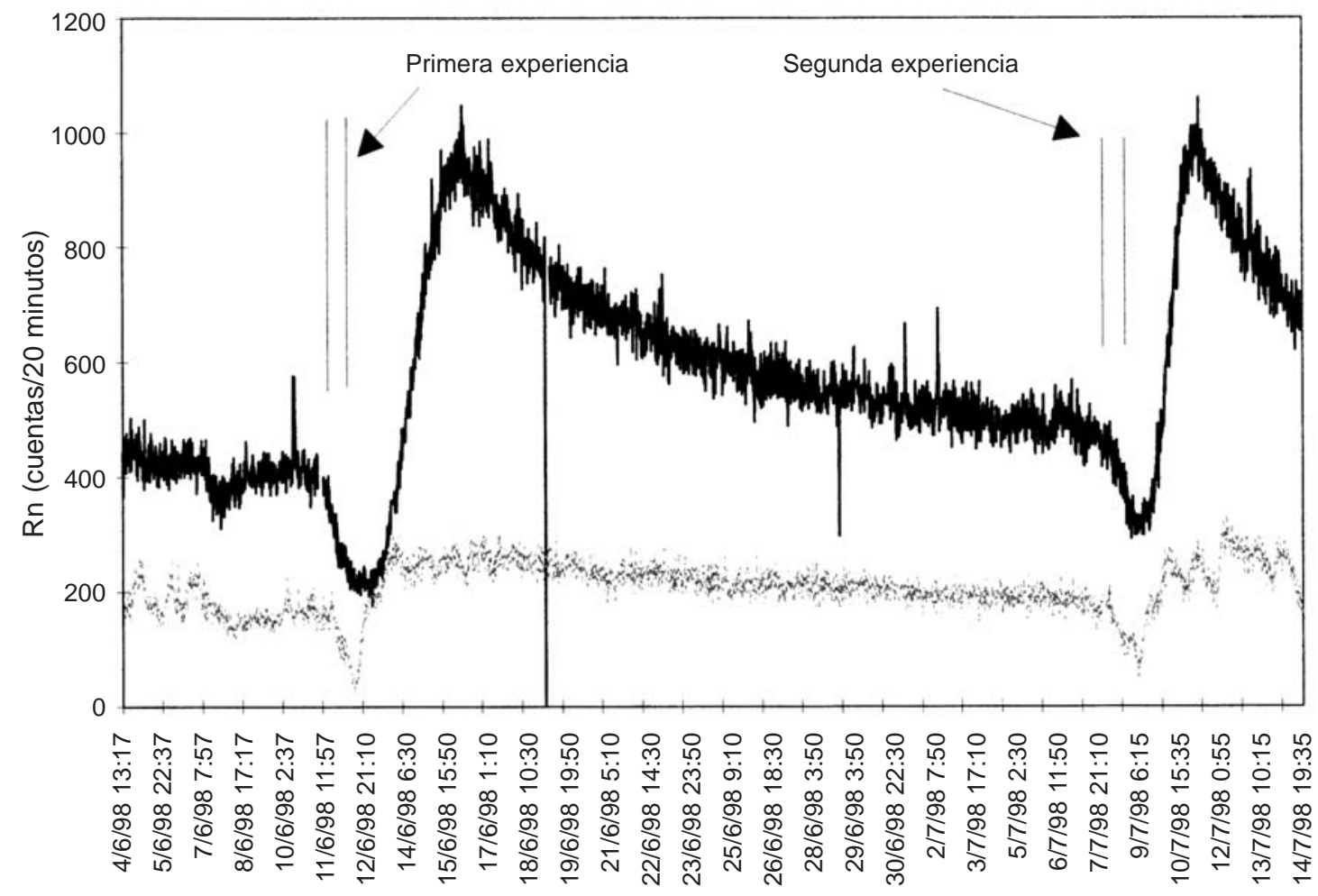

tiempo

\section{Sonda $1 \quad$...................... Sonda 2}

Fig. 2: Evolución temporal de la concentración de radón en las dos sondas del dispositivo de simulación. Las líneas verticales marcan el inicio y el final de la simulación de la lluvia. 
no miscibles, de manera que el agua desplaza al radón de los poros del suelo. Este efecto es percibido en las dos sondas casi al mismo tiempo debido probablemente a la gran permeabilidad del medio. Al final de la aspersión el suelo se encuentra completamente saturado de agua y esta continúa filtrándose hacia abajo por la acción de la gravedad, lo que hace que la concentración de radón siga disminuyendo aún más. La disminución en la concentración de radón se prolonga hasta que el flujo de agua que circula por los poros, debido a la gravedad, finaliza. Terminado este proceso la concentración comienza a aumentar lentamente. Para explicar este último fenómeno debemos hacer referencia a la ecuación de transporte del radón en medio poroso. Esta se escribe en una dimensión como:

$$
\frac{\partial \mathrm{C}}{\partial \mathrm{t}}=\frac{\mathrm{D}}{\varepsilon} \frac{\partial^{2} \mathrm{C}}{\partial \mathrm{z}^{2}}-\frac{\mathrm{v}}{\varepsilon} \frac{\partial \mathrm{C}}{\partial \mathrm{z}}-\lambda \mathrm{C}+\mathrm{Q}
$$

Donde C representa la concentración en $\mathrm{Bq} / \mathrm{m}^{3}$, D el coeficiente de difusión en $\mathrm{m}^{2} / \mathrm{s}$, $\varepsilon$ la porosidad, v la velocidad de Darcy en $\mathrm{m} / \mathrm{s}, \lambda$ la constante de desintegración del radón en $\mathrm{s}^{-1}$ y $\mathrm{Q}$ la tasa de producción de radón en $\mathrm{Bq} / \mathrm{m}^{3} \mathrm{~s}$. En nuestro caso la velocidad de Darcy es nula pues el sitio donde se encuentra el dispositivo está situado sobre una plataforma calcárea maciza a menos de dos metros de profundidad, típico del suelo mediterráneo, con lo que no hay contribución de radón por convección, proveniente de las capas profundas. De tal manera que el transporte está dado inicialmente por difusión solamente. La solución de esta ecuación describe la evolución temporal de la concentración en cada punto del sistema y debido a la simetría de nuestro dispositivo de simulación, la única variación espacial de la concentración se da con la profundidad.

Los coeficientes D y Q dependen fuertemente de la tasa de humedad del medio (Fleisher \& Mogro-Campero, 1978; Singh \& Virk, 1996; Fleisher, 1997). D puede variar, para un suelo seco, entre $10^{-8} \mathrm{~m}^{2} / \mathrm{s}$ hasta $10^{-5} \mathrm{~m}^{2} / \mathrm{s}$ y para un suelo húmedo su valor puede caer por debajo de $10^{-9} \mathrm{~m}^{2} / \mathrm{s}$ que es el coeficiente de difusión de radón en el agua. La tasa de producción $\mathrm{Q}$ viene dada como:

$$
\mathrm{Q}=\mathrm{E}_{\mathrm{Rn}} \rho \frac{1-\varepsilon}{\varepsilon} \mathrm{A}_{\mathrm{Ra}} \lambda
$$

$\mathrm{E}_{\mathrm{Rn}}$ representa la tasa de emanación de la roca, o sea, el cociente entre el número de átomos de radón que escapan del grano hacia los poros y el número total de átomos producidos por la roca. Esta cantidad es función de la tasa de humedad del medio; $\rho$ es la densidad del suelo en $\mathrm{kg} / \mathrm{m}^{3}, \mathrm{~A}_{\mathrm{Ra}}$ es la concentración de radio en el suelo dada en $\mathrm{Bq} / \mathrm{kg}$.

La tasa de emanación $\left(E_{R n}\right)$ es más grande cuando existe agua en los poros pues, el átomo de radón es capturado por esta debido a que su recorrido libre medio en el agua es muy pequeño (Tanner, 1980; Rama \& Moore, 1984), mientras que si el fluido intersticial es gas, el átomo de radón producido en un grano tiene la probabilidad de alcanzar el grano vecino y ser capturado por este.

Volviendo al objeto de estudio, una vez que el flujo de agua cesa por completo en el interior del sistema de simulación, el agua residual retenida en el suelo (líquida o vapor) aumenta la tasa de emanación del suelo y disminuye el coeficiente de difusión, provocando un aumento local de la concentración. Este aumento cesa después que, naturalmente, la humedad del suelo disminuye de nuevo, para volver a su estado inicial. El coeficiente de difusión aumenta al mismo tiempo que la tasa de humedad disminuye y paralelamente la tasa de emanación disminuye también. El radón llega a circular más fácilmente y no existe ya acumulación local. Los niveles de concentración alcanzan su valor inicial de equilibrio.

La primera experiencia se realizó con el suelo seco pero, de la Figura 2 se observa que al inicio de la segunda experiencia los niveles de concentración, en las dos sondas, estaban por arriba de los niveles iniciales correspondientes a la primera experiencia. Esto significa que el grado de humedad del suelo es más elevado al principio de la segunda experiencia que al principio de la primera. El efecto de esta diferencia en las condiciones iniciales del suelo se refleja en el comportamiento del radón. La disminución de la concentración, que se presenta al inicio de esta segunda experiencia, en las dos sondas, es menos intensa que la correspondiente a la primera. Sin 
embargo, cuando la concentración aumenta, esta alcanza los mismos valores en cada sonda para las dos experiencias. Esta observación se puede extrapolar concluyendo que cuando el suelo presenta un grado de humedad importante, la disminución en la concentración llegará a ser menos intensa aún hasta el límite de percibir solamente el aumento de la concentración. Este fenómeno es debido a que el agua de la lluvia no puede circular con la misma facilidad que cuando el suelo está seco o ligeramente húmedo. En este caso el agua permanece en las capas superficiales del suelo formando una barrera para el radón y aumentando así la concentración en las capas inferiores debido a la acumulación del radón.

La Figura 3 muestra un conjunto de mediciones de la concentración de radón tomadas a lo largo de un año. Estas mediciones, al igual que las mostradas en las figuras 4, 5 y 6 fueron realizadas en el campus de la Universidad de Costa Rica, específicamente en los jardines del Laboratorio de Física Nuclear, mediante sondas electrónicas tipo Clipperton (Monnin \& Seidel, 1998). De la figura 3 se aprecia que los valores de la concentración en la segunda mitad del año son superiores a los de la primera mitad. Este fenómeno está asociado con la precipitación presente en estos últimos meses del año. El aumento en la humedad del suelo debido a la precipitación es la principal causa de las variaciones de la emanación. Las figuras 4 y 5 muestran la evolución de la concentración del radón y de la precipitación en varios períodos.

Los datos de la figura 4 corresponden al inicio y mediados de la estación lluviosa. El suelo se encuentra parcialmente húmedo debido a las primeras lluvias. En esta figura se constata lo observado por nuestro experimento y el comportamiento del gas radón están de acuerdo con nuestro modelo. A cada precipitación se le asocia una disminución en la concentración del radón y luego un aumento significativo de esta, como lo predice el modelo.

La figura 5 muestra la evolución de la concentración de radón en período lluvioso. En este período el suelo se encuentra con una humedad importante debido a las constantes lluvias. El comportamiento del gas radón verifica

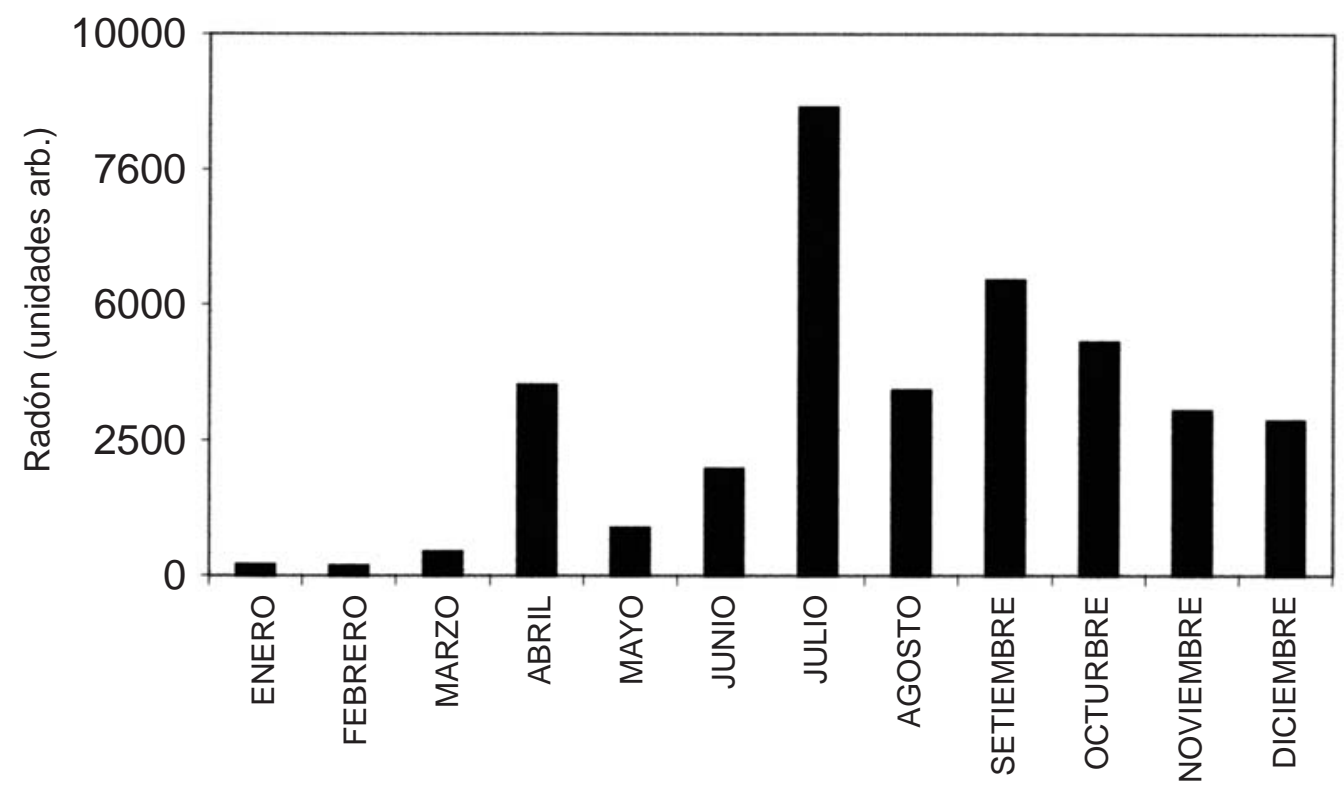

Fig. 3: Valores promedios de la concentración de radón durante un período de un año. 


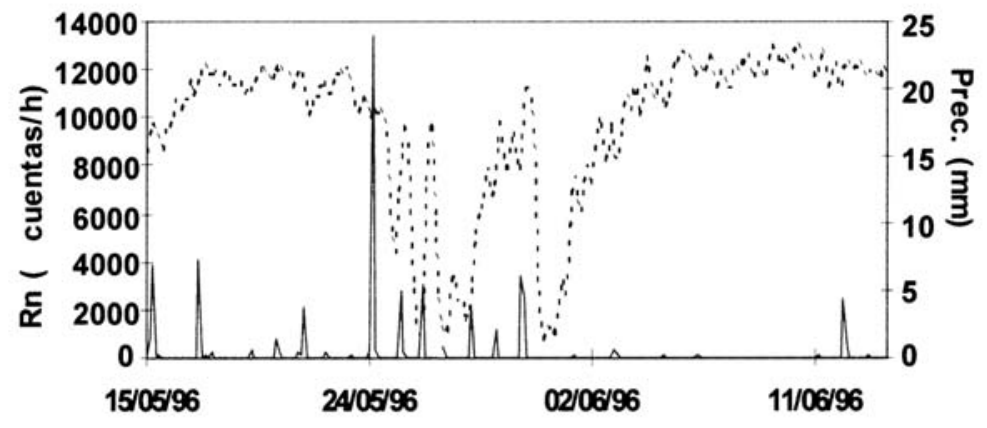

$\mathbf{a}$ -

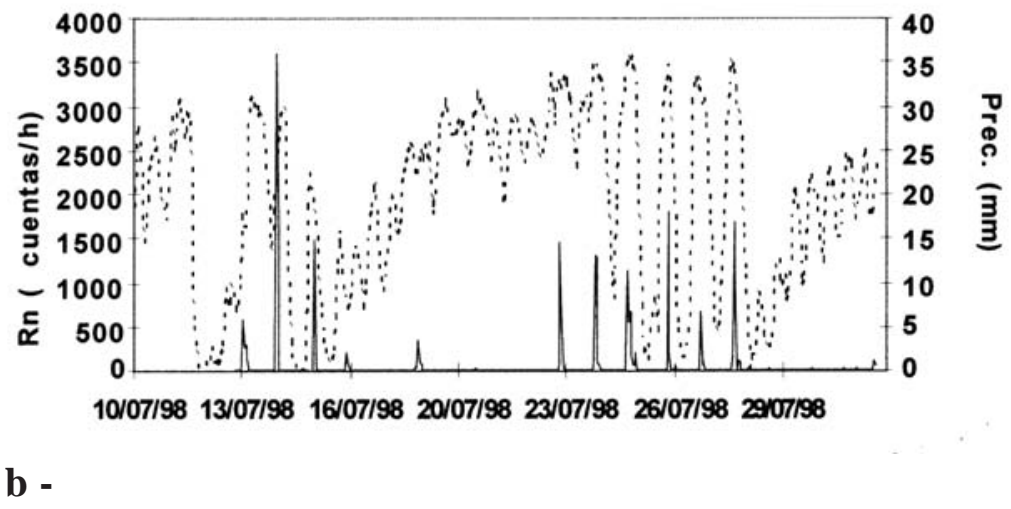

Fig. 4: Evolución de la concentración de radón y de la precipitación a) al inicio de la estación lluviosa y b) a mediados de esta, para dos años diferentes (radón en línea punteada).

la extrapolación realizada a partir de nuestro modelo. La concentración del gas aumenta bruscamente cuando se presenta una precipitación. La disminución inicial de la concentración, presente cuando el suelo esta seco o parcialmente húmedo, desaparece como lo predice el modelo.

Por último, la figura 6 muestra la evolución de la concentración del radón y la precipitación en época seca. En esta figura se observa claramente que las variaciones de la concentración de radón en ausencia de lluvia son puramente estadísticas, además el comportamiento seguido por el radón a partir del 16 de febrero concuerda con los resultados de nuestro experimento y lo predicho por el modelo, cuando los niveles de humedad del suelo son bajos.

\section{CONCLUSIONES}

Como se muestra en la sección anterior, tanto el experimento como el modelo propuesto coinciden con los datos de campo. Del modelo propuesto y de las experiencias realizadas podemos inferir que cuando tratamos con un suelo seco, como por ejemplo durante la estación de verano en los países temperados o la estación seca en los países tropicales, una lluvia fuerte podría provocar inicialmente una disminución de la concentración de radón en las capas superficiales del suelo debido a los fenómenos de disolución y transporte del radón por el agua de lluvia. En ausencia de lluvia durante las horas o días sucesivos, la humedad retenida por el suelo produce un 


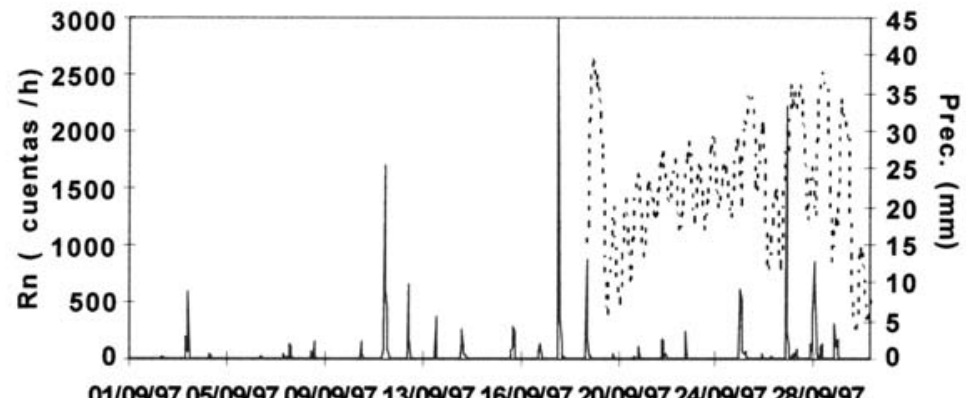

a -

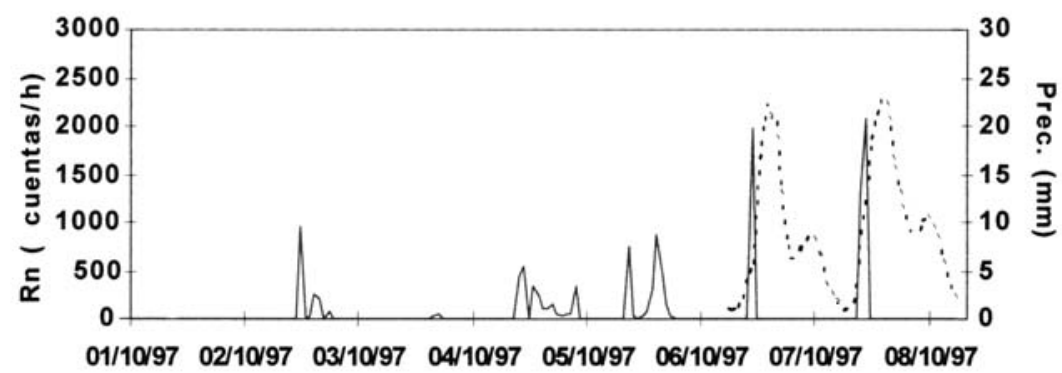

b -

Fig. 5: Evolución de la concentración de radón y de la precipitación en plena estación lluviosa del año 1997 (radón en línea punteada).

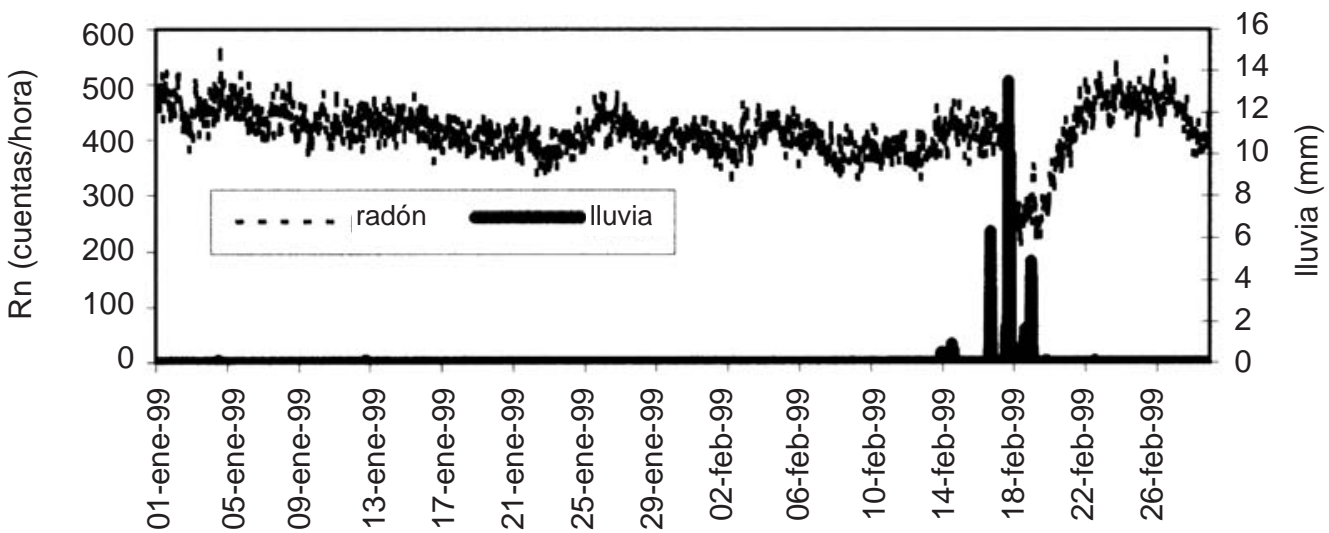

Fecha

Fig. 6: Evolución de la concentración de radón y de la precipitación en época seca. 
aumento de la tasa de emanación del radón y en consecuencia de la concentración de este en el suelo. Seguido de esto el radón deberá alcanzar los niveles normales de concentración, conforme la humedad retenida desaparece gradualmente.

Si la lluvia persiste, como en otoño e invierno en los países temperados o durante la estación lluviosa en los países tropicales, el suelo puede alcanzar niveles de humedad próximos al de saturación y en este caso el agua de lluvia permanece en las capas superiores del suelo, constituyendo una barrera que impide que el radón se escape hacia la atmósfera. Estos efectos pueden variar en intensidad según las propiedades del suelo (porosidad, permeabilidad, etc.), pero el mecanismo general es el mismo que el explicado en este trabajo.

Los resultados obtenidos y el modelo presentado en este trabajo son de gran utilidad a la hora de la interpretación de las medidas de radón en zonas volcánicas y sísmicas. Los resultados podrían dar lugar a la creación de un filtro para la influencia de la lluvia en las medidas realizadas en zonas de interés sísmico y volcánico.

Un análisis más cuantitativo de este fenómeno toma en cuenta la ecuación de transporte de radón y la ecuación de velocidad del frente húmedo, con el fin de poder obtener un flujo convectivo de radón. Este análisis forma parte de un futuro trabajo del autor.

\section{AGRADECIMIENTOS}

El autor agradece a la Universidad de Costa Rica, en especial a la Vicerrectoría de Investigación, por el apoyo brindado, a través del proyecto "Radón y Sismicidad en Costa Rica". A la Universidad de Montpellier II e igualmente al Organismo Internacional de Energía Atómica.

\section{REFERENCIAS}

ABBAD, S., ROBE, M.C., BERNAT, M. \& LABED, V., 1996: Influence of meteorological and geological parameter variables on the concentration of radon in soil gases: application to seismic forecasting in the Provence-Alpes-Cote d'Azur region. - Environ. Geochem Health, 16: 35-48.

CLEMENTS, W. \& WILKENING, M., 1974: Atmospheric pressure effects on Rn222 transport across the Earth-Air interface. J. Geophys. Res. 29: 5025-5029.

COTTER, J.M., 1990: Simulated transport of radon in soil gas. - 154 págs. University of Hawaii [Tesis MSc.].

FLEISCHER, R.L. \& MOGRO-CAMPERO, A., 1978: Mapping of integrated radon emanation for detection of long-distance migration of gases within the earth: Techniques and principles. - J. Geophys. Res. 83: 3539-3549.

FLEISCHER, R.L., 1997: Radon: Overview of properties, origin, and transport. - En: DURRANI, S.A., \& ILIC, R.(eds): Radon measurements by etched track detectors: Applications in radiation protection, earth sciences and the environment: 3-20. Saeed A. Durrani \& Radomir Ilic, World Sci. Publ. Singapore.

GARCIA-VINDAS, J. R., 1999: Transport du radon en milieu poreux (experimentation et modelisation) implication pour la realisation et l'interpretation de mesures in situ. 271 págs. Université de Montpellier, Francia [Tesis PhD.].

HINKLE, M.E., 1994: Environmental conditions affecting concentrations of $\mathrm{He}, \mathrm{CO} 2, \mathrm{O} 2$ and N2 in soil gases. - Appl. Geochem. 9: 53-63.

KING, C.Y., 1992: Comment on Rn222 premonitory signals for Earthquakes. - EOS, 73: 517-518.

KLUSMAN, R.W. \& JAACKS, J.A., 1987: Environmental influences upon Mercury, 
Radon and Helium concentrations in soil gases at site near Denver, Colorado. - J. Geochem. Explor. 27: 259-280.

LORÍA, L., BRENES, J., FERNANDEZ, E., JIMÉNEZ, R., GARCIA, R., MONNIN, M., SEIDEL, J.L., SEGOVIA, N. \& BALCAZAR, M., 1995: Radon and seismicity in Costa Rica. - Environ. Geochem. Health, 16: 213-219.

MOGRO-CAMPERO, A. \& FLESHIER, R.L., 1977: Subterrestrial fluid convection: a hypothesis for long-distance migration of radon within the Earth. - Earth \& Planet. Sci. Lett. 34: 321-325.

MONNIN, M.M. \& SEIDEL, J.L., 1992: Radon in soil-air in groundwater related to major geophysical events: A survey . - Nuclear Instruments and methods in Phys. Res. 314: 316-330.

MONNIN, M.M. \& SEIDEL, J.L., 1998: An automatic radon probe for earth science studies. - J. Appl. Geophys. 39: 209-220.

PAPASTEFANOU, C., MANOLOPOULOU, M., SAVVIDES, E. \& CHARALAMBOUS, S., 1989: Radon monitoring at Stivos Fault following the $\mathrm{ML}=6.5$ earthquake occurred at Thessaloniki Greece in 20 June 1978. - Nucl. Geophys. 3: 49-56.

PEARSON, J.E. \& JONES, G. E., 1965: Emanation of Radon 222 from soils and its use as a tracer. - J. Geophys. Res.20: 5279-5289.
PEARSON, J.E. \& JONES, G.E., 1966: Soil concentrations of "emanating radium 226" and the emanation of radon 222 from soils and plants. - Tellus, 18: 655-661.

PINAULT, J.L. \& BAUBRON, J.C., 1997: Signal processing of diurnal and semidiurnal variations in radon and atmospheric pressure: A new tool for accurate in situ measurement of soil gas velocity pressure gradient and tortuosity. - J. Geophys. Res.102(B8): 18101-18120.

RAMA, \& MOORE, W.S., 1984: Mechanism of transport of U-Th series radioisotopes from solids into ground water. - Geochem. Cosmochim. Acta, 48: 395-399.

RUDAKOV, V.P., 1985: Nature of the seasonal variations in subsoil radon. - Geokhimiya, 7: 1055-1058.

RUDAKOV, V.P., 1985b: Basic variations in subsoil radon. - Geokhimiya, 1: 124-127.

SINGH, B. \& VIRK, H.S., 1996: Effect of soil and sand moisture content on radon diffusion using plastic track etched detector. Radiat. Meas. 26: 49-50.

TALWANI, P., MOORE, W.S. \& CHIANG, J., 1980: Radon anomalies and microearthquakes at Lake Jocassee, South Carolina. J. Geophys. Res. 85: 3079-3088.

TANNER, A.B., 1980: Radon migration in the ground: A supplementary review. - Nat. Rad. Env. 3: 5-37. 